\title{
An assessment of environmental contamination arising from the use of some automated equipment in microbiology
}

\author{
GJ HARPER \\ From the Environmental Microbiology and Safety Reference Laboratory, Public Health Laboratory Service, \\ Centre for Applied Microbiology and Research, Porton Down, Salisbury, Wiltshire
}

SUMMARY The generation of aerosols and the contamination of surfaces arising from some equipment used to automate tasks in the microbiology laboratory have been assessed using a microbiological test method. When used for the designed purposes none of the equipment is likely to present any significant aerosol hazard. Some of the equipment gave rise to surface contamination, and it is recommended that decontamination of work surfaces should be carried out at frequent intervals.

Equipment to automate repetitive tasks is commonplace in biochemical and haematology laboratories. Similar equipment is now being introduced into clinical microbiology laboratories for the identification of micro-organisms, measurement of sensitivity to antibiotics, urine screening, and monitoring of blood cultures using a variety of techniques. Although Newsom and Matthews' showed that some automated equipment used for biochemistry was not likely to present a hazard from aerosols, no comparable assessment has been reported for equipment designed for microbiology.

The equipment can be divided into two groups. Systems such as the Autobac (General Diagnostics), Bactomatic (Medical Technology Corporation), and the Malthus (Malthus Instruments) are not likely to give rise to environmental contamination as the inoculation of the closed culture vessels in these systems is done using conventional techniques. In the second group, the subject of this report, mechanical systems are used to transfer infected or potentially infected fluids (either the primary specimen or a suspension of bacteria) or for the repeated sampling of the head space of culture vials. As it is normal practice to operate this equipment on the open bench, tests have been done to assess any contamination of the environment that might arise from their use.

A biological tracer, spores of Bacillus globigii, was used to measure aerosol generation and surface contamination in and around the equipment.

Accepted for publication 12 April 1984

\section{Material and methods}

EQUIPMENT TESTED

1 Sceptor System (Becton Dickinson)

2 Sensititre Automatic Inoculator (Seward

Laboratory)

3 Cobas Bact Analyser (Roche Products Ltd)

4 TDX Analyser (Abbott Laboratories Ltd)

5 Bactec 460 (Becton Dickinson)

\section{TESTING PROCEDURE}

The equipment was assembled, tested, and operated in accordance with the instruction manuals provided by the manufacturers.

Tests were done using methods previously described in a modified' Porton Class III microbiological safety cabinet; ${ }^{2}$ where the equipment was too large to be accommodated in the cabinet tests were done in a small test room $\left(23 \mathrm{~m}^{3}\right)$ fitted with HEPA filtered air supply and exhaust and ultraviolet lamps. Air samples were collected as close to the equipment as possible (see below) and surfaces sampled using sterile cotton wool swabs moistened with sterile water. Control samples of air and from the surfaces were collected before each test to measure any background contamination with the test micro-organisms. The test chamber was decontaminated with formaldehyde between each test and thoroughly aired. When the test room was used it was irradiated with ultraviolet light between tests. 


\section{AIR SAMPLING EQUIPMENT}

\section{Large volume glass cyclone}

This device, similar to that described by Decker et al, ${ }^{3}$ concentrates the particles from about 0.7 $\mathrm{m}^{3} / \mathrm{min}$ of air into $1 \mathrm{ml}$ of sterile phosphate buffer.

\section{Slit samples}

Particles are collected on to the surface of nutrient agar at a sampling rate of $0.025 \mathrm{~m}^{3} / \mathrm{min}$.

\section{Biological tracer cells}

Spores of $B$ globigii (NCT C No 10073) were added to all the test fluids to yield a final concentration of about $1 \times 10^{9}$ viable spores per millilitre. The test fluids used were not the same for all the equipment; details are given in the Results section.

\section{ASSESSMENT OF SAMPLES}

All cultures were made on the surface of plates of tryptose soya broth agar incubated before use to check sterility. Cyclone samples and suspensions, diluted where necessary in sterile phosphate buffer, were inoculated in measured volumes on to the surface of at least four plates. Swabs were rubbed over the surface of similar plates. Slit sampler plates were incubated without further processing. After incubation at $37^{\circ} \mathrm{C}$ for 18 to $24 \mathrm{~h}$, colony counts were recorded as colony forming units (CFU).

\section{Results}

Because of the diversity of the equipment tested, each item will be described briefly and reported on separately.

\section{SCEPTOR}

This is a semiautomated system for the identification of micro-organisms and for measuring the sensitivity of isolates to antibiotics. The system consists of an automated preparation station, single use test panels in which the material under test is incubated, a reader recorder, and a data management centre. The only part of the system that could conceivably give rise to microbial contamination of the environment is the preparation station. This is a microprocessor controlled unit for the rehydration and inoculation of the test panels with a broth culture containing about $1 \times 10^{5}$ micro-organisms per millilitre. A moving platform carries the test panel, which contains dried reagents in 84 wells, to a position below the delivery nozzle of a plastic reservoir containing $10 \mathrm{ml}$ of the suspension being tested. The reservoir is covered by a sealing cap with an $\mathrm{O}$ ring seal and the delivery nozzle is closed by a metal clamp. A small pump generates a positive pressure in the sealed head space over the reservoir. Material is automatically dispensed by pressure when the nozzle sealing clamp is opened under the control of the microprocessor. After purging, to ensure the dispensing nozzle is filled, $100 \mu \mathrm{l}$ volumes of suspension are delivered to each well in the test panel. Any fluid remaining in the reservoir is pumped into a beaker containing disinfectant in a waste drawer under the working deck. This process takes about one minute. A fresh reservoir and test panel are used for each organism tested.

For the tests the reservoir was filled with $10 \mathrm{ml}$ of Sceptor broth containing $B$ globigii cells. Air samples were collected during the dispensing cycle (including the pouring of the inoculum into the reservoir) and when opening the lid. This was repeated with three test panels on two occasions, making a total of six tests. Surface swabs were collected before and after each set of three tests. Between each set of tests the working deck was decontaminated with buffered glutaraldehyde followed by water and then dried.

\section{Aerosol generation}

No particles containing $B$ globigii were collected by slit samplers placed on either side of the preparation station in any of the tests. A cyclone sampler recovered small numbers of $B$ globigii during dispensing in two tests and when the lid was opened at the end of the dispensing cycle in one test. The highest recovery of $B$ globigii, $108 \mathrm{CFU}$, is equivalent to the cells contained in $0 \cdot 1$ nanolitres of the suspension added to the reservoir, during the dispensing of $10 \mathrm{ml}$ into one test panel.

\section{Surface contamination}

$B$ globigii were recovered from the working deck under the dispensing nozzle and from a swab collected from the panel platform. All other sites swabbed were negative for $B$ globigii.

\section{SENSITITRE}

This is designed to carry out tasks similar to the Sceptor. The system consists of an automated inoculator, single use test plates, a plate viewer reader, and a data interpretation unit. Only the automatic inoculator was examined. The function of this is to dose 96 well microtitre plates with a measured volume of a broth suspension containing about $1 \times 10^{5}$ micro-organisms per millilitre. A broth culture is added to a glass tube fitted with a single use plastic dosing head. The tube is inverted and attached to the dispensing head of the inoculator, where the inoculum is dispensed automatically into the wells of a microtitre plate attached to a moving carrier. Dispensing is done by a piston with a synchronised push-pull and rotary action. Retraction of 
the piston fills the dispensing cylinder, a twisting motion opens an outlet valve, and a forward movement dispenses $50 \mu \mathrm{l}$ of inoculum through the nozzle of the dosing head. A second twist of the piston head returns the valve to the inlet position and the process is repeated. The dispensing mechanism, dosing pattern, and movement of the microtitre plate are controlled by a microprocessor. Although the inoculator is designed to operate in five different modes, the instrument supplied was programmed for use in only two. Mode 1 dispenses $50 \mu$ l to all 96 wells in a plate in about $35 \mathrm{~s}$. In Mode 2 two $50 \mu \mathrm{l}$ volumes are dispensed into each of 24 wells. When used in Mode 2 four suspensions can be tested using a single plate.

For each test, $10 \mathrm{ml}$ of a suspension of $B$ globigii in water was dispensed into a single plate. In Mode 1 three plates were dosed in rapid succession with a change of inoculum tube between plates. In the Mode 2 tests each plate was filled by carrying out four 24 well cycles from one inoculum tube. Air sampling was continuous throughout each test and sampling continued for several minutes after the last plate had been filled. The equipment was decontaminated by exposure to formaldehyde between tests. Four tests were carried out using each of the operating modes.

\section{Aerosol generation}

All the air samples collected during the operation of the inoculator in Mode 1 were free from $B$ globigii. In the Mode 2 tests small numbers of $B$ globigii were recovered by both cyclone and slit samplers. The highest recovery, $50 \mathrm{CFU}$, is equivalent to the cells contained in 0.05 nanolitre of the test suspension collected during the dispensing of $28.8 \mathrm{ml}$ into three microtitre plates. Slit sampler plates showed a maximum of 2 CFU in similar circumstances.

\section{Surface contamination}

No $B$ globigii were recovered from the microtitre plate carrier in any of the tests. Swabs collected from the tray under the dispensing head, the bridge carrying the dispenser, the dispensing head, and the surface of microtitre plates yielded $B$ globigii on some occasions. Recovery was more frequent (19/28 swabs positive) when the inoculator was operated in Mode 2 than in Mode 1 , where $6 / 26$ swabs yielded $B$ globigii.

\section{COBAS BACT ANALYSER}

This is a completely automatic system for the identification of micro-organisms and for carrying out antibiotic sensitivity tests in sealed disposable plastic rotors.

Each rotor contains 16 tests wells spaced round the perimeter and a larger central well to hold the inoculum. The tests wells of the rotor are loaded with discs containing dried reagents and the wells closed with a plastic sealing ring. Inoculation of the rotor is carried out by pipetting $5 \mathrm{ml}$ of a broth culture into the central well, which is then sealed with a self adhesive label. The inoculated rotor is placed in the loading position of the Cobas Bact Analyser. From this point, the rotor is handled automatically by a microprocessor controlled system.

The rotor is raised to engage with the underside of the centrifuge head and a $300 \mu$ l volume of the inoculum is transferred to each of the test wells by centrifugal force. The rotor is then transported to an incubator compartment in which it is kept agitated. At 20 min intervals the rotor is transferred from the incubator to the centrifuge head. After a brief period of agitation to suspend the contents of the test wells, the rotor is turned so that each well passes under a light sensor. After reading, the rotor is returned to the incubator compartment. This process is repeated 15 times over a period of $5 \mathrm{~h}$, after which the rotor is discarded into a waste drawer under the working platform. The results are displayed by a thermal printer. For each completed test, the rotor is subjected to 16 cycles of centrifugation. To speed up the testing procedure the analyser was operated using Programme 120, the Quick Mode. This process transfers the rotor from the incubator to the centrifuge head at intervals of $24 \mathrm{~s}$ in place of the $20 \mathrm{~min}$ used in the normal operational programme, allowing a test to be completed in $6 \mathrm{~min}$.

For each test a rotor was loaded with $5 \mathrm{ml}$ of Cobas Bact broth containing $B$ globigii. Normally the centrifuge head is surrounded by a metal shield For three of the tests this shield was removed to see if it played any part in reducing environmental contamination. Air sampling was carried out during the 6 min needed for the complete cycle of transfer and centrifugation. A total of nine tests were carried out. No surface decontamination was done between tests, but the room and equipment were irradiated with ultraviolet light.

\section{Aerosol generation}

On three occasions low concentrations of $\boldsymbol{B}$ globigii were measured in control air samples collected before the tests (maximum $20 \mathrm{CFU}$ in a cyclone; 3 CFU by slit sampling). Recoveries of $B$ globigii during these tests were similar to the control values. In the other six tests no $B$ globigii were recovered in either control or test samples. In one test the exterior of a rotor was inadvertently contaminated during loading. The use of this rotor and the removal of the shield normally placed round the 
centrifuge head did not result in the recovery of any airborne particles.

\section{Surface contamination}

With the exception of a swab from the known contaminated rotor, all the surfaces sampled were free from $B$ globigii in all tests.

\section{TDX ANALYSER}

This is an automated system to monitor the concentrations of drugs, hormones, and antibiotics in serum or plasma using fluorescence polarisation immunoassay. As serum samples being assayed for their antibiotic concentrations could contain viable micro-organisms, the process was examined using serum containing $B$ globigii.

Samples for assay are pipetted manually into the outer well of disposable plastic sample cartridges, which are held in a 20 place carousel. The analyser, which is controlled by a microprocessor, automatically pipettes the sample while simultaneously pipetting buffer into the dilution well of the sample cartridge. Reagents are then added from a reagent pack housed alongside the carousel, and the mixture of diluted sample and reagents is finally transferred to a glass cuvette. Each cuvette is then positioned in front of a sensor and the results printed. The time needed to process 20 samples is about $10 \mathrm{~min}$. All pipetting operations are made using a probe attached to a boom, which moves in an arc from the reagent pack to the carousel; the probe is automatically raised and lowered as required. Normally the TDX can be operated only with the front panel closed. To allow air samples to be collected as close as possible to the working area the electronic lock was overridden during the tests and the front panel kept in the open position. On three occasions a carousel containing 20 sample cartridges was loaded by pipetting a $200 \mu \mathrm{l}$ volume of horse serum containing $B$ globigii into the sample well of each cartridge. The loaded carousel was placed in position in the TDX and the assay process started by pressing the RUN control button. Air samples were collected throughout the operating cycle and for $4 \mathrm{~min}$ after the assay had finished. No surface decontamination was carried out between tests, with the exception of the probe, which was cleaned with water and wiped dry with a paper towel as laid down in the manufacturer's operations manual. The room and equipment were irradiated with ultraviolet light between tests.

\section{Aerosol generation}

None of the air samples collected with the cyclone during assays contained $B$ globigii in excess of the low numbers recovered during the control sampling periods $(<10 \mathrm{CFU})$. In two tests a slit sampler col- lecting directly over the cuvettes showed the presence of a single CFU. No $B$ globigii were recovered by a slit sampler positioned over the sample cartridge.

\section{Surface contamination}

With the exception of the pipetting probe, no $B$ globigii were recovered from the surfaces swabbed. A single water wash of the probe followed by drying with a paper towel was sufficient to reduce contamination to an undetectable level on two occasions and to a very low level on the third occasion.

\section{BACTEC 460}

This differs from the rest of the equipment tested. The Bactec is used to test sealed vials of culture media for evidence of bacterial growth by monitoring the gas in the head space for ${ }^{14} \mathrm{CO}_{2}$ derived from ${ }^{14} \mathrm{C}$ labelled subtrates in the media. It is used for monitoring blood cultures for growth at regular intervals.

Vials are sampled after cleaning the exposed rubber septa with alcohol to eliminate the risk of contamination. Two needles, sterilised by an electric heater immediately before use, are inserted through the septum into the head space of the sealed vial. A sample of gas is drawn by means of a vacuum pump through an ion counter for measurement of radioactivity. The gas withdrawn from the vial is replaced with sterile gas from a storage cylinder. When loaded with a batch of vials (the instrument can accommodate up to 60 ), each vial is positioned automatically under the sampling head, the needles are sterilised and allowed to cool, and the head space gas sampled. This process is repeated until all the vials have been sampled. Results are displayed visually and recorded by a printer.

The Bactec is designed to be operated with the transparent lid in the closed position. Failure to do so may affect the needle steriliser and impair the correct functioning of the system. To allow air samples to be collected as close as possible to the sampling station the equipment was used in these tests with the lid fixed in the open position by overriding the safety interlock. The needle steriliser was seen to operate correctly between each sampling operation.

On each of three days a batch of six Bactec Aerobic Culture Vials No 6 was inoculated with $B$ globigii to yield a final concentration of about $6 \times$ $10^{8} \mathrm{ml}$. Although the manufacturers' manual recommends that alcohol should be used to decontaminate Bactec vial caps, a preliminary swabbing with $5 \%(\mathrm{vol} / \mathrm{vol})$ chloros was carried out to reduce contamination with the hardy test spore. This was 
followed by washing with water and swabbing with alcohol.

The inoculated vials were shaken on a reciprocal platform for $10 \mathrm{~min}$ and inverted once to simulate the procedure given in the Bactec manual. The vials were then placed in the Bactec and sampled on two occasions in tests 1 and 2 and three times in test 3 . In addition to collecting air samples throughout the vial sampling process, settle plates (open petri dishes containing culture media) were placed close to the sampling station. The sampling needles were removed and autoclaved after each test. No other surface decontamination was done. The room and equipment were irradiated with ultraviolet light between tests.

\section{Aerosol generation}

No $B$ globigii were recovered in any of the air samples collected during these tests.

\section{Surface contamination}

Swabs from the rubber septa of six of the 18 vials used yielded small numbers of $B$ globigii $(<5 \mathrm{CFU})$ before sampling in the Bactec. This probably reflects incomplete decontamination after inoculation of the vials with concentrated $\boldsymbol{B}$ globigii suspension. After sampling in the Bactec on either two or three occasions, swabs from 12 of the vials showed the presence of $B$ globigii greatly in excess of the small numbers found in some of the pre-test samples. All the other sites swabbed and the settle plates were negative for $\boldsymbol{B}$ globigii.

\section{Discussion}

The concentration of tracer cells in the fluids used in these tests, about $1 \times 10^{9} / \mathrm{ml}$, is greatly in excess of the numbers likely to be found in material normally processed in the equipment. This was done to increase the sensitivity of the tests and allow extrapolation to the lower numbers usually encountered.

In practice, the recommended concentration of cells for use in the Septor, Sensititre, and Cobas Bact is of the order of $1 \times 10^{5} / \mathrm{ml}$. In the TDX analyser much lower numbers would be expected in serum or plasma being examined for antibiotic concentrations. With the Bactec, initial levels of viable cells in blood cultures are likely to be low but will increase as growth takes place in the culture vials. However, samples will be recorded as positive at cell populations well below the levels used in these tests.

Slit samplers collecting at a rate of $50 \mathrm{l} / \mathrm{min}$, several times greater than the respiration rate of man, were usually free from colonies of the tracer organism. The highest recovery with this sampler was 2 CFU during the dosing of three microtitre plates using the Sensititre in Mode 2 . When we examined the recoveries from the cyclone the maximum recovery was equivalent to the number of cells contained in $0 \cdot 1$ nanolitres of suspension during the dispensing of $10 \mathrm{ml}$ using the Sceptor.

If we use the maximum value found with the cyclone sampler to assess the likely consequences when dispensing materials containing $1 \times 10^{5}$ cells $/ \mathrm{ml}$, then the numbers of cells that would be found in an aerosol would be of the order of 0.001 cell per millilitre dispensed. Clearly, none of the equipment tested can be regarded as hazardous as a result of aerosol generation when used with the range and concentrations of micro-organisms likely to be present in materials being handled in a clinical microbiology laboratory. This is in agreement with the findings of Newsom and Matthews ${ }^{1}$ when they assessed the aerosol hazards from some automated equipment used in biochemistry.

Swabs from some of the surfaces in and around the equipment were contaminated with tracer cells, the Sceptor and Sensititre showing the heaviest contamination. No surface contamination was detected with the Cobas Bact even when it was used with an externally contaminated rotor. The pipetting probe of the TDX was readily decontaminated by following the manufacturer's instructions. The finding of contamination in the rubber septa of Bactec culture vials after sampling suggests that in addition to being cleansed before each sampling it would be prudent to decontaminate the vial tops after sampling.

It is reasonable to assume that in normal usage surface contamination will be at a much lower level that that found in these tests, which used highly concentrated suspensions. Nevertheless, it is a sound policy to decontaminate the surface of automated equipment at frequent intervals to obviate transfer of contamination to the operator or other parts of the laboratory.

I am grateful to Becton Dickinson, Seward Laboratories, Laboratory Impex Ltd, Roche Products Ltd, and Abbott Laboratories Ltd for loaning the equipment used in this work.

\section{References}

' Newsom SWB, Matthews J. A microbiological survey of automated biochemical machines. J Clin Pathol 1980;33:566-70.

${ }^{2}$ Harper GJ. Contamination of the environment by special purpose centrifuges used in clinical laboratories. $J$ Clin Pathol 1981;34:1114-23.

${ }^{3}$ Decker HM, Buchanan LM, Frisque DE, Filler ME, Dahlgren CM. Advances in large volume air sampling. Contamination Control 1969;8:13-7.

Requests for reprints to: Mr GJ Harper, PHLS Centre for Applied Microbiology and Research, Environmental Microbiology and Safety Reference Laboratory, Porton Down, Salisbury, Wiltshire SP4 0JG, England. 